\title{
3D monitoring for plant growth parameters in field with a single camera by multi-view approach
}

\author{
Yu Zhang ${ }^{a}$, Poching Teng ${ }^{a}$, Mitsuko Aono ${ }^{b}$, Yo Shimizu ${ }^{a}$, Fumiki Hosol ${ }^{a}$ and Kenji Omasa ${ }^{a}, \dagger$ \\ $\left.\begin{array}{c}{ }^{\mathrm{a}} \text { Graduate School of Agricultural and Life Sciences, The University of Tokyo, 1-1-1 Yayoi, Bunkyo-ku, Tokyo 113-8657, Japan } \\ { }^{b} \text { National Institute for Environmental Studies, 16-2 Onogawa, Tsukuba-city, Ibaraki 305-8506, Japan }\end{array}\right)$
}

\begin{abstract}
In recent decades, some photogrammetric methods for 3D monitoring of plant growth and structure parameters have been studied by the automated feature extraction and matching. In this study on growth analysis of sweet potato (Ipomoea batatas L.) plants at different fertilizer conditions, we proposed a convenient solution of 3D reconstruction by a single camera photography system based on Structure from Motion (SfM) method. Also, we handled effectively the noise problem by minimizing re-projection errors. The results of $3 \mathrm{D}$ models demonstrated that the average percentage error was a constant about $4.8 \%$ for plant height or decreased from $13 \%$ to $8 \%$ (leaf area index, LAI $=3.5$ ) for leaf number and from $19 \%$ to $12 \%(\mathrm{LAI}=3.5)$ for leaf area with increasing in $\mathrm{LAI}$, although each percentage error fluctuated, especially at low LAI. In contrast, the average percentage error in 2D image processing was $20 \%$ to $45 \%$ for leaf number and $60 \%$ to $90 \%$ for leaf area, and the leaf height was immeasurable. Comparing with the errors of $3 \mathrm{D}$ results, the errors of $2 \mathrm{D}$ images were much larger because $2 \mathrm{D}$ imaging had some problems, such as not being robust against occlusion of plant organs, and the ambiguity between object size and distance from the camera. On the other hand, we examined a method to calibrate the estimates from 3D models using the regression model between the measured value and the value estimated from 3D model. The regression models showed the linear and good estimation for leaf height $\left(\mathrm{R}^{2}=0.97\right.$ and $\left.\mathrm{RMSE}=0.71 \mathrm{~cm}\right)$, leaf number $\left(\mathrm{R}^{2}=0.99\right.$ and $\left.\mathrm{RMSE}=4.03\right)$ and leaf area $\left(\mathrm{R}^{2}=0.98\right.$ and RMSE $=0.12 \mathrm{~m}^{2}$ ), in spite of use of data across a wide range in fertilizer supply and growth stage of sweet potato plants. The results demonstrated that $3 \mathrm{D}$ imaging technique in the study has the potential to remotely monitor plant growth status and estimate growth and structure parameters at various environmental factors outdoors.
\end{abstract}

Key words: 3D modeling, Leaf area, Leaf number, Plant height, SfM

\section{Introduction}

The importance of plant growth and structure parameters in estimating crop yield and controlling the microclimate is widely recognized, but the measurements of plant growth and structure parameters are challenging, time consuming, and often rely on destructive methods. Over the past three decades, several studies about plant growth and structure parameters monitoring were conducted, which are non-destructive, automated, image-based measurement technologies (Omasa et al., 2007; Lati et al., 2013; Muller-Linow et al., 2015). Over the past decades, 2D imaging has been applied ranging from such growth analysis, structural analysis, environment assessment, and yield estimation at different growth level or different period (Omasa, 1990; Se et al., 2012; Li et al., 2014). However, compared with 3D models, 2D imaging has a number of shortcomings (Gonzalez and Woods, 2008; Li et al., 2014), such as not being robust against occlusion of plant organs because of many overlapping leaves and stems, not being able to obtain fully 3D information because of complex 3D plants structure, and the ambiguity between object size and distance from the camera.

Thus, the application of $3 \mathrm{D}$ imaging technologies has

Received; March 8, 2018

Accepted; July 17, 2018

†Corresponding Author: aomasa@mail.ecc.u-tokyo.ac.jp

DOI: 10.2480/agrmet.D-18-00013 gradually been a long-time challenging study topic. In recent decades, 3D imaging technologies are being widely applied in plant science and modern agriculture (Snavely et al., 2006; Omasa et al., 2007; Kazmi et al., 2014; Zhang et al., 2016). With the development of 3D imaging and the improvement of sensor accuracy, this research area is now receiving more attention than ever before. $3 \mathrm{D}$ imaging techniques include contact and non-contact methods. During the last decade, some non-contact optical imaging methods like passive-based and active-based $3 \mathrm{D}$ reconstruction methods have been developed and improved for a wide range of applications, such as LiDAR or Kinect system based on structured light (Omasa et al., 2003, 2007; Hosoi et al., 2011; Lou et al., 2014), and the digital camera photography system based on stereovision (Rovira-Más et al., 2005; Paproki et al., 2012; Tippetts et al., 2013; Rose et al., 2015). The advantages of active methods like LiDAR lie in not only the measurability of homogeneous objects but also the canopy penetration while their disadvantages include the prohibitive cost and being easily influenced by wind movement outdoors (Hosoi et al., 2011; Rose et al., 2015). On the other hand, the advantages of passive methods include lower cost, higher resolution and more detailed estimates. But passive methods are usually time consuming when it comes to image processing. Comparing passive methods with active methods, self-occlusion and correspondence problems are common. Generally speaking, passive methods are applied more and more to fields with the specific advantages attributed to their low-cost 
and higher resolution, and especially practicality value in multi-view observation for plant growth and structure parameters monitoring. They may also improve the self-occlusion and correspondence problems.

In the passive methods, a common approach of 3D imaging technology, such as stereovision, shape from focus/shading/texture, structure from motion etc is normally applied (Omasa and Kouda, 1998; Se et al., 2012; Rose et al., 2015; Nguyen et al., 2016; Teng et al., 2016; Zhang et al., 2016). The most widespread method of acquiring 3D data is stereovision. Stereovision provides high resolution depth data with stereo views comparing with active methods but it is constrained by the texture of the plants along with computational efficiency. Self-occlusion and correspondence problems are also its problems. As for shape from focus/shading/texture using a single camera, the method is simple and it avoids complex stereo correspondence with single view resulting in problems of self-occlusion and correspondence. Comparing with the above methods, Structure from Motion (SfM) has more advantages. It can handle large number of views, minimize self-occlusion problem and optimize correspondence problem. However there also exist the problems of noises and data miss. Besides, the processing time may take a lot of time with the growing of views number. In general, SfM is becoming an effective method for 3D reconstruction with high-resolution because of its advantages of low-cost, and solving self-occlusion and correspondence problems.

In recent years, computer vision has successfully employed SfM techniques on multi-view imagery for the generation of high-resolution 3D models such as digital surface models (DSM) and digital elevation models (DEM). SfM can be applied to large collections of overlapping images to obtain point clouds for a wide range of objects, such as buildings and sculptures. The power of this technique was demonstrated by Snavely et al. (2006) who used the Bundler software to reconstruct 3D models based on hundreds of overlapping images available from a photogrammetric camera. SfM technique is based on identifying matching features in images taken from different views. For example, multi-camera photography system was developed to effectively capture images from different views and reconstruct fine-scale 3D models by Zhang et al. (2016). Image features are identified by scale invariant feature transform (SIFT) algorithm (Lowe, 2004), which is robust in terms of its feature descriptors for image features at different viewing angles.

More recently, there have been several aboveground studies on $3 \mathrm{D}$ reconstruction of plant growth and structure parameters. These studies have successfully demonstrated the use of SfM for generation of high-resolution 3D point clouds and surface models from a photogrammetric technique of single camera. For example, Santos et al. (2014) adopted a kind of computer-aided image acquisition method by freely moving the single camera around the plants to get overlapping images from different views to solve occlusions problem. The presented approach relies on local features detected on the plant surface. But the lack of feature points in some plants can make camera localization unfeasible. Experiments with maize specimens were unsuccessful because of presenting almost featureless surfaces. Rose et al. (2015) conducted the image acquisition with a Canon EOS 450D camera. The study did not include extracting additional morphological parameters, like leaf length/number/area, leaf area index (LAI) and stem diameter. The limit of detectable details should be studied, as well. Jay et al. (2015) retrieved the crop row 3D structure with a single digital camera. The camera calibration parameters could be automatically estimated from the data by SfM. But the method did not consider occluded leaves and further investigation is needed to retrieve leaf area for such plant structures. Besides, the wind-related problem is also a major obstacle in the field as the plants may not be in the same position from one image to the other, which would lead to poorer reconstruction results.

In this research, the previous problems will be addressed in our experiment by SfM and re-projection error minimization algorithm. The algorithm is conducted by optimizing the location of 3D points and the camera. The efficiency and accuracy of the proposed method were assessed at difference growth stages and culture conditions as well as its robustness against various plant structures and acquisition techniques outdoors. The objectives of this study are to not only explore a highly-effective data capturing method outdoors and 3D reconstruction method from multi-view images by SfM but also to optimize the 3D modeling method by accuracy assessment between measurement results and estimation results based on plant growth and structure parameters. In sum, the overall purpose is to build high-resolution 3D plants models from multi-view overlapping images by applying an improved SfM technique and relating it to growth parameters measurements of sweet potato plants in the field, aiming at accurate retrieval of plant growth and structure parameters based on 3D plants models. Finally, we hope to provide the quantification assessments and monitoring solutions for plant growth and structure parameters by $3 \mathrm{D}$ imaging technologies during different growth periods.

\section{Materials and Methods}

\subsection{Study area}

The study was conducted at the farmland of National Institute for Environmental Studies Ecosystem Research Field II $\left(36.05^{\circ} \mathrm{N}, 140.08^{\circ} \mathrm{E}\right)$ in Tsukuba, Japan (Fig. 1). The study farmland is $60 \mathrm{~m}$ by $20 \mathrm{~m}$ including 23 rows of sweet potato (Ipomoea batatas L.) plants with approximately 125-140 plants per row. Figure 1A shows the overview of study area and the fertilizer amount in the divided 6 sample areas, leading to desirable variation in the plant height, canopy structure and leaf area across the site. The study farmland is equally divided into six sample areas by red line and labeled in numbers (S1 to S6). The area of each sample area is $10 \mathrm{~m} \times 20 \mathrm{~m}$. Figure 1B shows the side view of one sample block in S1 sample area; including sweet potato plants (green), plastic mulch film (black), and soil within the area. Figure $1 \mathrm{C}$ shows the young sweet potato plants of one sample block $\left(1 \times 1 \mathrm{~m}^{2}\right)$ in $\mathrm{S} 1$ sample area. Each sample block is fenced up by four ferric pillars on 4 corners and a white string.

We chose sweet potato plants as our study object. We transplanted the seedlings into the farmland in April 2015 and started to monitor them and take images one or two times every month since May 2015. We only measured two sweet potato plants of one sample block $\left(1 \times 1 \mathrm{~m}^{2}\right)$ for every sample area each 
time. The actual measurement parameters include leaf number, leaf area, and plant height and so on. For the direct measurements of leaf number and area, we adopted destructive way that means cutting them and calculating their number or area. As for the plant height, we used a ruler scale by hand. Besides, there are different fertilizer treatments in the six sample areas (Fig. 1A). The fertilizer compound contained 3\% fertilizer, 10\% Phosphoric Acid, 10\% Potassium Kinsei Kasei No.3, Taki Chemical Co., Japan and 2\% Granular Magnesia (Tagen Lime Industry Co., Japan). The fertilizer amount in S1 to S6 sample area $\left(200 \mathrm{~m}^{2}\right)$ is $0 \mathrm{~kg}\left(0 \mathrm{~kg} / \mathrm{m}^{2}\right), \quad 5 \mathrm{~kg} \quad\left(0.025 \mathrm{~kg} / \mathrm{m}^{2}\right), 10 \mathrm{~kg} \quad\left(0.05 \mathrm{~kg} / \mathrm{m}^{2}\right)$, $20 \mathrm{~kg}\left(0.1 \mathrm{~kg} / \mathrm{m}^{2}\right), 30 \mathrm{~kg}\left(0.15 \mathrm{~kg} / \mathrm{m}^{2}\right)$ and $40 \mathrm{~kg}\left(0.2 \mathrm{~kg} / \mathrm{m}^{2}\right)$, respectively. Uniform fertilization was carefully conducted to keep spatial uniformity of plant growth in each area.

\subsection{Image collection}

For this study, a moderately priced, consumer-grade digital single lens reflex (SLR) camera (EOS Kiss X7, Canon Industrial Co., Ltd, Japan) with a Cannon GPS GP-E2 receiver was used. This camera is equipped with an approximately 1800 megapixel-( $5184 \times 3456$ pixels $)$ CMOS sensor with ISO range of 100 to 12800 . The focal length of the lens used was $28 \mathrm{~mm}(\mathrm{f} / 1.8)$ and a polarization filter (Circular PL for reflection removal, Kenko Tokina Japan Co., Ltd, Japan) was added to the lens. Before capturing the image, we calibrated the camera for lens distortion compensation.

In our experiment, we have chosen a $28 \mathrm{~mm}$ lens for the field experiment. The number of view positions of taking images were totally 53 views with this single camera whose purpose is to make the overlapping images cover the study block (see Fig. 2). The overlap ratio is over $90 \%$. The camera took an image with GPS position information in every view. The images were taken with 8 bit JPEG resolution by the camera. Time required for one measurement was about 8 minutes. These image datasets will be input into the PhotoScan (Agisoft LLC, St. Petersburg, Russia) for calibration processing and $3 \mathrm{D}$ model building in the computer (see Fig. 3).

All images were collected from 10 June 2015 to 19 October 2015. As for data acquisition during different periods, some references (the red-and-white pillar and the cubic, etc) would be laid across the study area for referencing purposes and independent accuracy assessment. Especially after 13 July, the plants grew lushly and needed the estimation references. Ground control points (GCPs) could be easily identified on the collected imagery. Their exact locations were measured directly after image acquisition in real-time kinematic mode, providing
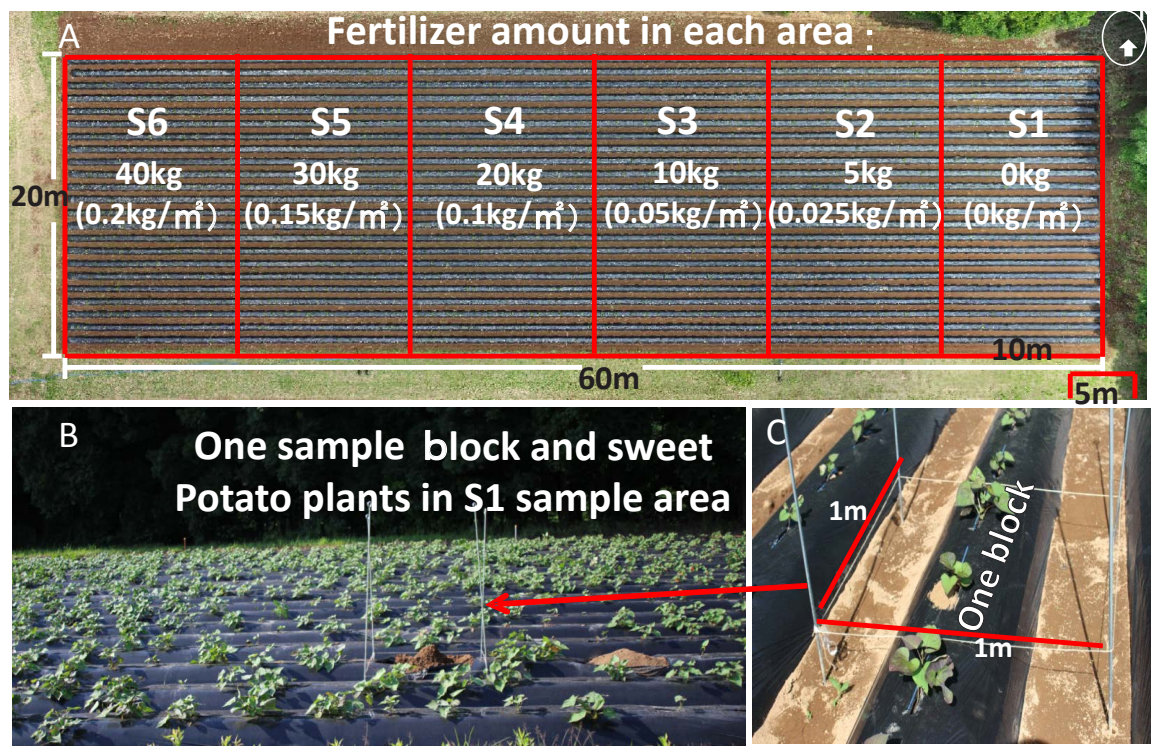

Fig. 1. The scheme of six sample areas: (A) The overview of study area and the fertilizer amount in the divided 6 sample areas; (B) The side view of one sample block $\left(1 \times 1 \mathrm{~m}^{2}\right)$ in S1 sample area; (C) The young sweet potato plants of one sample block in S1 sample area taken in June 10, 2015.

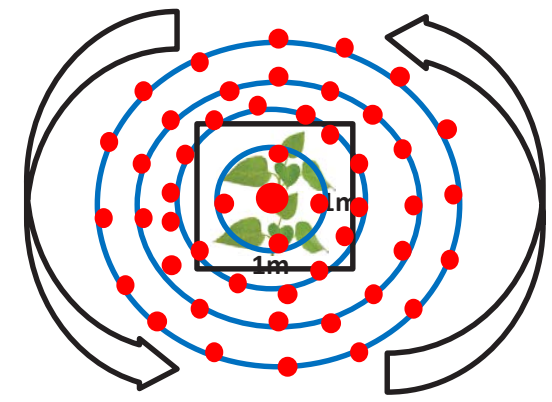

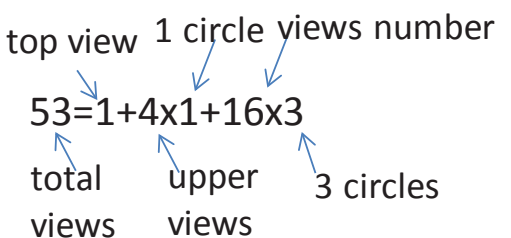

Fig. 2. Conceptual diagram of view positions of taking images with a single camera. The number of view positions of taking images is 53 views. The overlap ratio is over $90 \%$. 
centimeter positional and height accuracies. Finally, the original images and generated 3D models were visually assessed on the basis of quality, viewing angle, and overlap, in order to remove blurred and under- or over-exposed images by the re-projection error minimization algorithm. In addition, we also performed image processing of $2 \mathrm{D}$ images including leaf number and leaf area by classifying image color with maximum likelihood and calculating leaf parameters (see in Fig. 3). Then we compared the accuracy between estimated and measured values for $2 \mathrm{D}$ images and 3D models.

\subsection{D modeling of plants}

$\mathrm{SfM}$ is an effective method for 3D modeling, which is the process of inferring $3 \mathrm{D}$ structure of a static scene from multi-view images. The conventional SfM method is applied for the estimation of 3D camera motion and structure (Oliensis, 2000; Carrivick et al., 2016). The method can be used to obtain 3D data of objects and calibrate a cluster of camera positions and 3D point's locations for each camera track (Leberl et al., 2010; Turner et al., 2012; Mathews and Jensen, 2013).

In SfM method, the uncalibrated original image data were processed and calculated by PhotoScan. As was shown in Fig. 3, there are 6 steps of image processing during the 3D rebuilding. The image data taken from 53 views had individual coordinate systems. First of all, in order to make them have the same 3D coordinate system, the image datasets obtained from 53 views were co-registered into the orthogonal coordinates and aligned by location identification.

As for the estimated matching points and camera positions, the datasets were registered through an accurate conversion. Then the conversion was repeatedly extracted by selecting in turn matching points from the image dataset and getting the best translation and rotation matrices that minimize an error estimation on account of the distance among them. This process was conducted for all couples of image dataset. Then the colored point clouds of stereo image data captured were produced by the SfM algorithms after calibrating the camera. These points are often located by $\mathrm{X}, \mathrm{Y}$, and $\mathrm{Z}$ coordinates in $3 \mathrm{D}$ coordinate system. The SfM algorithm starts from the calibrated stereo images and a set of tracks. For properly calibrated camera positions, undistortion of stereo images is actually combined with rectification. The process computed disparity images in turn from incoming stereo images using the block matching algorithm. The disparity images were generated using a local correlation algorithm based on Sum of Squared Differences (SSD). It was optimized for efficiency and had a number of validation steps to prune disparity. Note that the extracted 3D point clouds were entangled with redundancies, a large number of outliers and noise. Fortunately, the additional information provided by the calibrated images can be exploited to help later mesh and surface reconstruction. After exporting 3D points data, dense point clouds model was reconstructed in four steps, which includes calculating depth information for each camera position, capturing dense point clouds correspondences, reconstructing structure from motion and 3D dense point clouds. The dense point clouds could be edited and classified or exported to an external tool for further analysis. Then, the classified dense point clouds of plants were transformed into polygon meshes. This procedure did some basic filtering to eliminate the most obviously irrelevant points. Thereafter, such polygon mesh images of plants were allowed to calculate the area of leaves.

Besides, during 3D modeling of plants, there are some noises

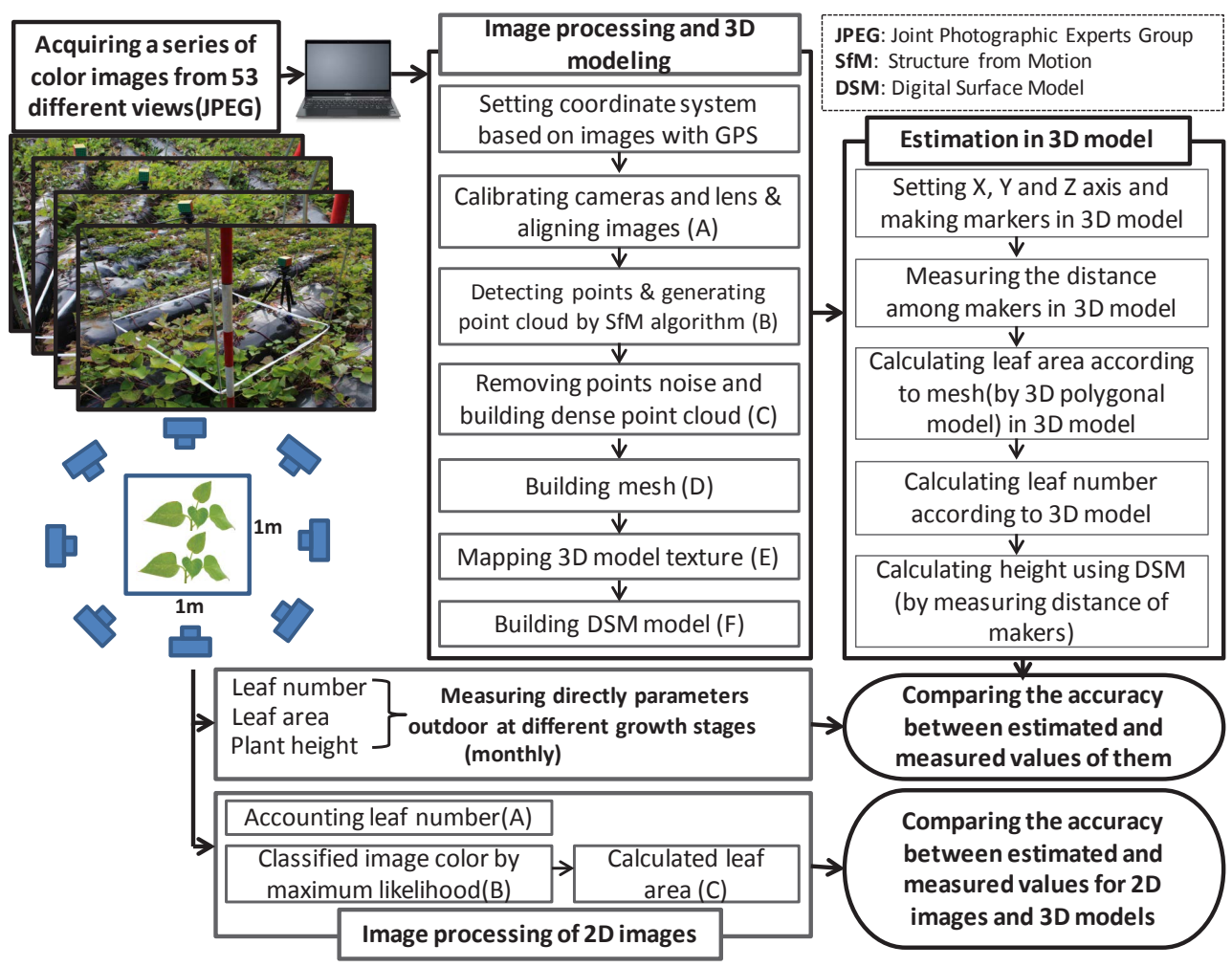

Fig. 3. The flowchart for 3D modeling and measurement. 
in plant leaf model. These noises increase with leaves growing more and larger. They were resulted from the overlapping of lush leaves, incorrect estimation of the feature points and color extraction in segmentation of surroundings. Thus, SfM method is not very accurate in feature detection and $3 \mathrm{D}$ modeling of plants. In order to minimize and mitigate self-occlusion and correspondence problem effectively, the re-projection error minimization algorithm was developed to improve image consistency with high-accuracy. We use estimate (E), projection matrix (M), 3D points $(\mathrm{S})$ and $\mathrm{W}$ as combined matrix.

$$
\mathrm{E}(\mathrm{M}, \mathrm{S})=\sum_{i=1}^{m} \sum_{j=1}^{n} \mathrm{~W}\left(\mathrm{~s}_{i j}, \mathrm{M}_{i} \mathrm{~S}_{j}\right)^{2}, \quad i=1, \ldots, m ; j=1, \ldots, n .
$$

According to the algorithm, the leaf with noise problem was detected and removed effectively. Besides, to reduce image analysis errors mentioned previously, it is necessary to include leaf shape analysis for taking the overlapping leaf areas into account and improve the system calibration algorithm for increasing the search accuracy of target positions.

Therefore, 3D surface texture of plant height and leaves was expressed as the inconsistent triangle meshes depending specially on alignment of point cloud after noise exclusion. Moreover, triangulated meshing and surface reconstruction were applied to generate surface texture models. Laplacian smoothing was employed to smooth triangle meshing. A series of natural color images captured after mesh were matched on the surface model of the entire plant using a texture-mapping approach. The texture-mapping approach is generally used as a method for putting surface texture into a computer-reconstructed 3D surface model (Koniaris et al., 2014). The 2D images like natural color images are available as the surface model reconstruction. Through the coordinate's correspondence of 2D image to $3 \mathrm{D}$ model, the feature of 2D image is automatically matched onto each mesh shell of the 3D model. A rigid choosing of the matching points between $2 \mathrm{D}$ and $3 \mathrm{D}$ images is very important to generate the complicated texture mapping of the sweet potato plants. Then the matching points were selected from each plant height and leaf. Whereafter, exact 3D natural color models were constructed. From the models, some information can be refined, such as plant height, leaf number and area, which are significative in plant detection and recognition.

\subsection{The measurements and estimates of plant growth and structure parameters in $3 \mathrm{D}$ model}

According to the above method, a 3D model is built and served as a basis for retrieving plant growth parameters. This model is computed using SfM method with RGB images acquired by translating a single camera. Then, to estimate the measurement parameters including leaf number, leaf area and plant height, plants and background are discriminated by a robust method that uses both color and height information in order to handle low-contrasted regions. The leaf number can be counted from 3D model. As for the calculation of leaf area in 3D model, each leaf polygon was automatically counted and estimated within leaf mesh. For the direct measurement of leaf area, we calculated leaf area by destructive method. We firstly cut all the leaves from sweet potato plants of 6 sample blocks and spread them on the desk to take the plane fanning leaves images after taking images in 6 sample blocks of 6 experiment areas each time. Then we could compute leaf area in Software LIA32 (an image analysis freeware for estimating LAI (Yamamoto, 2004)) according to the plane fanning leaves images. Plant height was calculated from stem tip to soil surface by calculating the distances of measured marked points according to the coordinates of referenced points in 3D model.

Besides, as an important ecophysiological parameter and plant structural variable in the description of many vegetation and plant growth processes, LAI was investigated. It is the total one sided green leaf area per unit ground area in broadleaf canopies, as seen in the Eq. (2). Many destructive and non-destructive methods have been developed to quantify LAI from the ground and describe other structural parameters. Knowledge of LAI variation during the whole plant cycle is essential for the modeling of plant growth and the estimation of yield (Clevers and Van Leeuwen, 1996). Some experiments have been carried out to estimate LAI and other biophysical parameters of vegetation and plants by optical remote sensing technology during the growing periods and for growth models and monitor plant growth (Hosoi et al., 2011). They have demonstrated that LAI is correlated with the fresh biomass of plants.

$$
\text { LAI }=\frac{\text { Leaf area }\left(\mathrm{m}^{2}\right)}{\text { Ground } \text { area }\left(\mathrm{m}^{2}\right)}, \quad \text { Ground area }=1 \times 1 \mathrm{~m}^{2} .
$$

In this study, LAI were measured and obtained within randomly selected areas of $1 \mathrm{~m} \times 1 \mathrm{~m}$. In order to monitor the growth conditions of the sweet potato plants, the relationship between LAI and other growth and structural parameters, such as height, leaf number and the error ratio, were also investigated. Consequently, the sweet potato plants were measured from June to October 2015 in each sample block. Data from 6 sample blocks with $1 \times 1 \mathrm{~m}^{2}$ area were shown in the corresponding measurements.

In the outdoor experiments, we have also surveyed GPS coordinates information on four pillars of 4 corners. In order to estimate the parameters of plants from 3D model, we used some references for accuracy estimation, such as the red-and-white pillar, the cubic and white rope. For example, one red-and-white pillar on one of four corners in each block was once served as the reference to set the referenced coordinates points during the latter measurements. The gap distance between red and white marker is $10 \mathrm{~cm}$. The coordinates in the pillars were set as reference coordinates with $3 \mathrm{D}$ coordinates for the assessment of the geometric accuracy, namely that westing $(\mathrm{X})$, southing $(\mathrm{Y})$, and height $(\mathrm{Z})$ was carried out for the orthomosaic. These referenced point clouds can be used to provide very specific and exact representations of a close depth scene. The coordinates of the key points were retrieved from the image according to the corresponding GPS coordinates, resulting in mean and root mean squared error (RMSE) accuracy measures in the $\mathrm{X}$ and $\mathrm{Y}$ direction. The height value was derived from the DSM for the GCP and also compared to the GPS observations, producing mean and RMSE accuracy measures for the Z direction (Höhle and Höhle, 2009; Harwin and Lucieer, 2012). Finally, we could obtain the direct and indirect measurement data. According to these data, we could calculate correlation coefficients $\left(\mathrm{R}^{2}\right)$ and 
RMSE between the estimated and measured values of these parameters to obtain their accuracy.

2.5 The measurements and estimates of growth and structure parameters in 2D images

In the past studies, 2D imaging had been applied ranging from such growth analysis, structural analysis, environment assessment, and yield estimation at different growth level or different period. Figure 4 showed the procedure example of measured parameters based on 2D image in S1 block at July 13,2015 , including leaf number and leaf area. It is the same for other blocks or other measurement periods. The original image might be considered to contain sub-images sometimes referred to as regions-of-interest (ROIs), or simply regions (see Fig. 4A). The original image could be considered to be a function of two real variables, for example, $\mathrm{a}(\mathrm{x}, \mathrm{y})$ with a as the amplitude (e.g. brightness) of the image at the real coordinate position $(\mathrm{x}, \mathrm{y})$. In order to obtain important information by identifying and analyzing $2 \mathrm{D}$ original image, the classification process that groups image pixels into categories was necessary. Firstly, we set RoIs and classify regions as different colors for plant, soil, string, pillar and vinyl with ENVI (Exelis VIS Co. Ltd.,2016).

Then, in Fig. 4B, the 2D original image was classified for training data by the supervised classification method. This method allows us to cluster pixels and pre-define classes, creating "training data" to work from when classifying the rest of the image. In this step, a supervised classification was performed where we provided training data and specified a classification method of maximum likelihood. The following determinant functions for each pixel in the image by Maximum Likelihood classification (MLC) (Richards, 2012) with supervised classification method were calculated:

$$
g_{i}(x)=\ln p\left(\omega_{i}\right)-1 / 2 \ln \left|\sum_{i}\right|-1 / 2\left(x-m_{i}\right)^{T} \sum_{i}^{-1}\left(x-m_{i}\right)
$$

Where,

$i=$ the $i$ th class

$x=\mathrm{n}$-dimensional data (where $n$ is the number of bands)

$g_{i}(x)=$ determinant functions for each pixel in the image

$p\left(\omega_{i}\right)=$ probability that a class occurs in the image and is assumed the same for all classes

$\left|\Sigma_{i}\right|=$ determinant of the covariance matrix of the data in a class $\Sigma_{i}-1=$ the inverse of the covariance matrix of a class

$m_{i}=$ mean ROI of a class

MLC assumes that the statistics for each class in each band is normally distributed and calculates the probability that a given
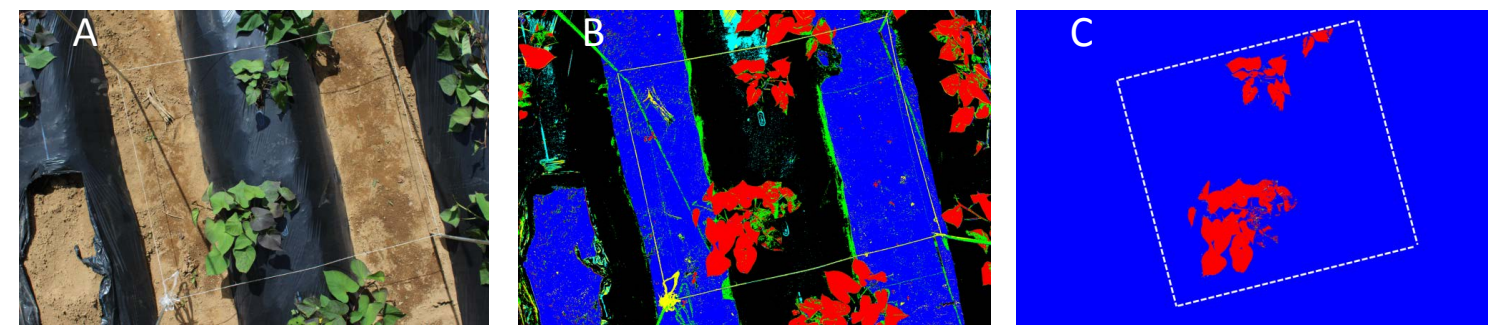

Fig. 4. The procedure example of measured parameters based on 2D image in 6 sample blocks at July 13, 2015: (A) Original image; (B) Classified image by the maximum likelihood method as a supervised classification from R(red), G(green) and $\mathrm{B}$ (blue) images of color photos; (C) Calculated leaf parameters (leaf number and leaf area) based on leaves left.
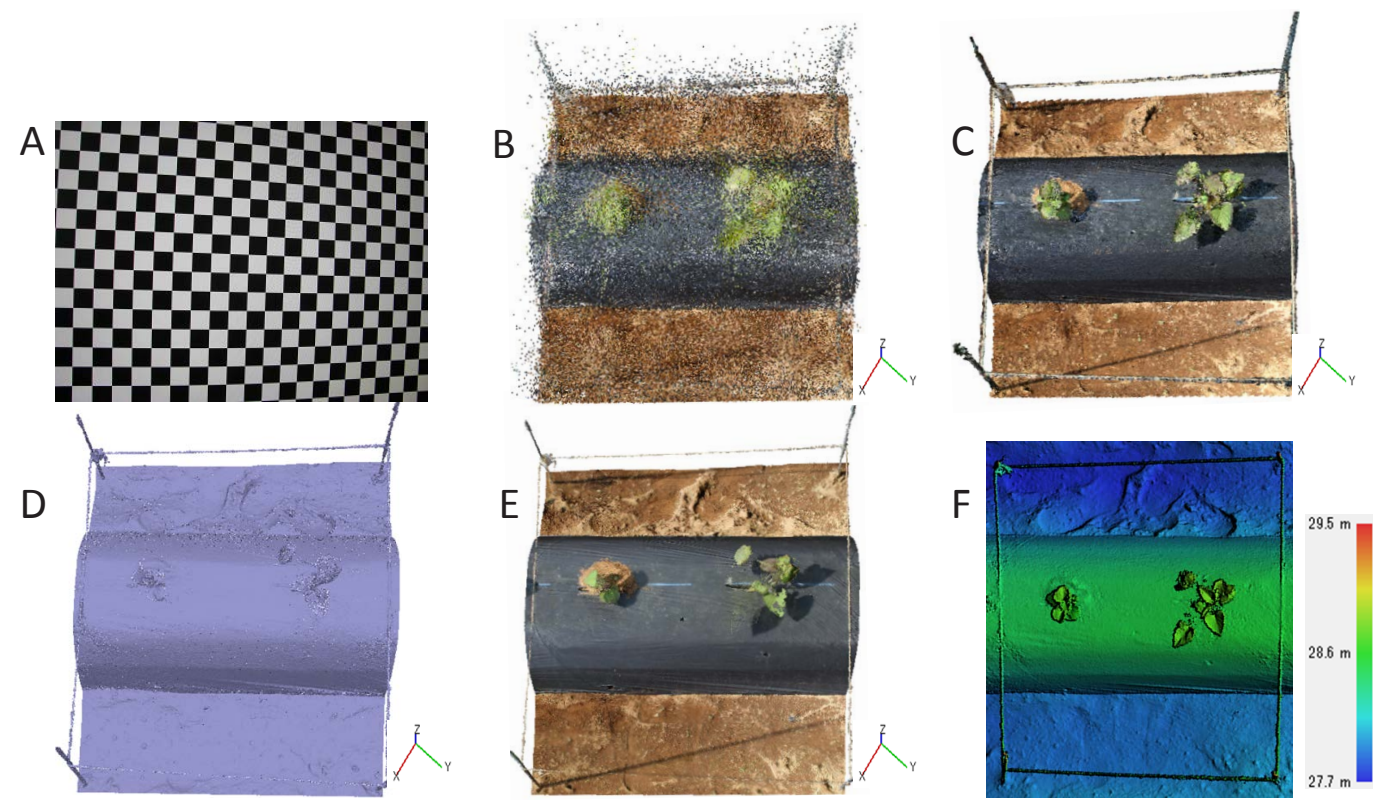

Fig. 5. The procedure for 3D modeling of sweet potato plants: (A) Camera and lens calibration; (B) Point cloud; (C) Dense point cloud; (D) Mesh (3D polygonal model); (E) 3D model texture; (F) DSM model. 
pixel belongs to a specific class. After dividing a 2D color photo to $\mathrm{R}$ (red), G(green) and B(blue) images, 2D RGB images were classified by the maximum likelihood method as a supervised classification and showed with different colors in Fig. 4B. After that, we could export classification results to an ENVI classification file. According to the results, we calculated plant growth parameters, such as leaf number and leaf area. For the counting of leaf number, we simply counted them on 2D RGB images. As for leaf area, Figure $3 \mathrm{C}$ is the estimated results of leaf parameters after calculating by Lia32. In this way, we were able to obtain the estimated accuracy of measured parameters for $2 \mathrm{D}$ image as well as the comparison between actual and estimated value in 2D image of parameters in 6 sample blocks at different growth periods.

\section{Results and discussion}

\subsection{D modeling of sweet potato plants}

Figure 5 shows an overview of 3D modeling procedure for sweet potato plants reconstructed from a series of images taken by the $28 \mathrm{~mm}$ lens. Firstly, Figure 5A shows camera and lens calibration in the internal parameters of the camera. Then, according to the process of 3D modeling shown in Fig. 3, PhotoScan generated point clouds with a total of 112,316 points after registration from the input images captured by the camera, as shown in Fig. 5B. Currently each point in the clouds is determined by the images and overlapping areas have a lot of points. It would be more accurate if this was determined by all possible images. It is best to define the minimum number of images for the determination of a point. Secondly, after removing the noise within the point cloud dataset, a total of 3,456,487 dense points remained in Fig. 5C. The dense point clouds gave greater precision, and its point number is thirty times more than one of the point clouds and much more accurate. Then, the dense clouds of plants were converted into the corresponding polygons mesh in Fig. 5D. It was seen that the polygons consist of many uneven triangle meshes. The complex curves and unevenness on the sweet potato plant model were generated on the polygon meshes. The number of polygon meshes per leaf was determined by unevenness polygon size in addition to leaf size. Figure 5E shows the texture mapping of 3D models from a series of images for sweet potato plants. In Fig. 5F, DSM models were reconstructed.
In sum, from Fig. 5F, we can see that most of leaves and stems shape were reconstructed and their clear models were generated from a series of images taken by the $28 \mathrm{~mm}$ lens.

Typically, during building the point clouds, noise also increased with increasing point density. The positions of some points were slightly higher or lower than actual model, which could not reproduce any actual character of $3 \mathrm{D}$ sweet potato plant model. Therefore, these points would be directly classified as noise points, most of which were removed during building the dense clouds next step. The filtered point clouds produced an accurate 3D modeling of the sweet potato plants as shown in Fig. 5E. Each leaf feature was clear because of the high-resolution images captured by our system.

\subsection{Growth and structure parameters of sweet potato plants measured by direct method}

In our experiment, a total of 12 plants were sampled at random in 6 sample areas of the field. We measured the growth of plant height, leaf number and leaf area by a single camera 6 times, from June to October 2015. The different fertilizer amount in the divided 6 sample areas led to desirable variation in the plant height, structure and leaf area across the sample areas. As is seen in Fig. 6, the plant growth rate in six sample areas S1 to S6 changed according to the different fertilizer treatments. The standard errors of growth parameters at each measurement were shown. The plant growth in area with more fertilizer treatments was significantly better than that in area with less fertilizer treatments. Especially, S5 and S6 sample blocks have the best growth state of leaf and stem biomass where the fertilizer amount is $30 \mathrm{~kg}$ and $40 \mathrm{~kg}$, namely $0.15 \mathrm{~kg}$ and $0.2 \mathrm{~kg}$ per $\mathrm{m}^{2}$.

Figure $6 \mathrm{~A}$ to $6 \mathrm{C}$ showed changes in the parameters with the growth of sweet potato plants. It illustrated the measurement results of these parameters were evaluated at sample blocks during different growth periods. For example, Figure 6A showed plant height ranged between $5 \mathrm{~cm}$ and $25 \mathrm{~cm}$ over all the growth periods of sweet potato plants. The plant height of S5 sample block was the highest in 10 August and the plant height of S6 sample block was the second highest in 5 September. This was because the plant height of S5 block could not continue to grow higher but grew longer until August. But the plant height of S6 block still continued to grow higher until September. Seasonal
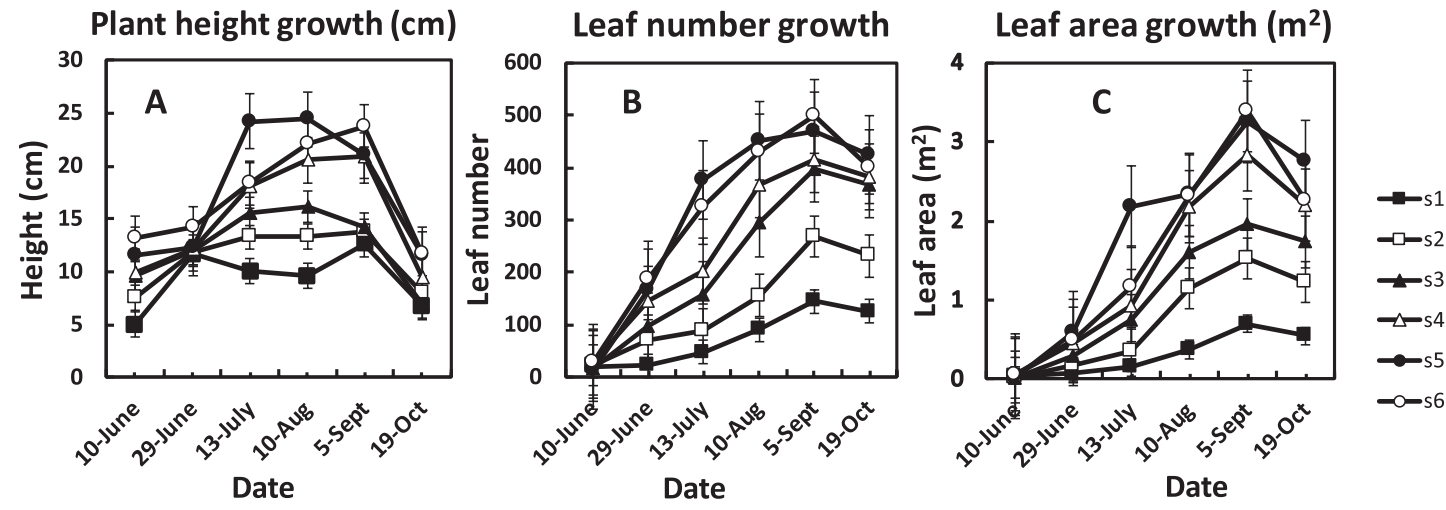

Fig. 6. The direct measurement of sweet potato plants growth parameters in 6 sample blocks (12 sample plants) at different growth periods from June to October 2015: (A) Plant height growth; (B) Leaf number growth; (C) Leaf area growth (Each point is the mean value and the vertical bar is standard error). 
growth change of plant height (Fig. 6A) is more complex than the other results (Fig. 6B and 6C), probably because plant height will be affected by various factors, such as effects of wind speed and light competition in adjacent individuals as well as the effect of fertilizer amount on each organ's growth. In Fig. 6B, the generated leaf number ranged between 5 and about 500 during the growth period. Only the leaf number of S6 sample block was the most in 29 June and 5 September. But the leaf number of S5 sample block was the most during other periods. S4 also continued to grow until September despite fertilizer amount is less than S5.

Besides, as the growth speed of sweet potato plants would change slower after September, the stem started to atrophy and leaf number changed less. So October should be the best harvest time. Likewise, Figure 6C also showed the increasing trend of leaf area as sweet potato plants grew and leaf number increased before 5 September. The value of leaf area ranged from $0.01 \mathrm{~m}^{2}$ on 10 June to $3.39 \mathrm{~m}^{2}$ on 5 September. Until October, S5 sample block had a better status with the most leaf number and the biggest leaf area. Thus, different fertilizer treatments had an important effect on the growth of sweet potato plants during its growth period. According to the measured results, Figure 6 demonstrated the fertilizer amount treatment with $0.15 \mathrm{~kg}$ per unit block was the most suitable for the growth of sweet potato plants.

In this study, we not only monitored the growth status of sweet potato plants during the growth periods but also obtained the data series of their structure parameters by a destructive method. As is seen in Fig. 6, we found the best fertilizer amount and fertilizer treatments for the growth of sweet potato plants. In general, according to the parameters measurement results of sweet potato plants, we can analyze the performance of different measurement periods in different sample areas. These analyses are very helpful for understanding the relationships between plant growth parameters and its environment such as light and temperature conditions, effects of fertilizer, irrigation and disease, etc.

\subsection{The relationship between LAI and the error of growth parameters estimated from 3D model}

As LAI is defined as the total one-sided leaf area per unit ground surface area is a growth indicator, we investigated the relationship between LAI and the error of growth parameters estimated from 3D model to the direct measured value. Figure 7 showed the relationship between LAI and percentage error of estimated parameters and the changing trend of the parameter error with the growth of sweet potato plants and increasing LAI in 6 sample blocks at different growth periods. The trend of a decreasing percentage error of estimated parameters as LAI increases can be observed. The value of LAI ranges from 0.1 to 3.5. The results of $3 \mathrm{D}$ models demonstrated that the errors linearly increased for plant height and leaf number and exponentially for leaf area with increasing in LAI, but the average percentage error shown in the regression line was a constant about $4.8 \%$ for plant height or decreased from $13 \%$ to $8 \%(\mathrm{LAI}=3.5)$ for leaf number and from $19 \%$ to $12 \%(\mathrm{LAI}=3.5)$ for leaf area with increasing in LAI, although each percentage error fluctuated, especially at low LAI (Fig. 7). These results may be insufficient for use in growth management and breeding in the farmland, hence we examined the regression models in next sections.

Furthemore, the percentage error largely fluctuated near LAI 0 in Fig. 7C. During the early periods of plant growth, sweet potato plants had few and small leaves. The absolute error of 3D model was easily affected by slight camera shake and wind in the field during the image capturing in addition of inherent spatial resolution of camera. On the other hand, the real measured value of plant leaves also has some error and the absolute error is constant. So, relatively, the percentage error is larger in the early periods of sweet potato plant growth.

\subsection{The relationship between LAI and the error of growth parameters estimated from 2D images}

For similarity with the estimation of growth parameters in 3D model, we also investigated the relationship between LAI and the absolute error of the growth parameters estimated from 2D images. As plant height was difficult to be estimated from the 2D image, we only made the estimation of leaf number and leaf area. Figure 8 shows the relationship between LAI and the percentage error of growth parameters estimated from 2D images in 6 sample blocks at different growth periods. From Fig. 8, we found the average percentage error was $20 \%$ to $45 \%(\mathrm{LAI}=3.5)$ for leaf number and $60 \%$ to $90 \%(\mathrm{LAI}=3.5)$ for leaf area, and leaf height was immeasurable. Comparing with the percentage errors estimated from 3D models, it was demonstrated that the percentage error from 2D images was very large. Comparing with the results in Fig. $7 \mathrm{~B}$ and $7 \mathrm{C}$, we found that the growth parameters values estimated from 3D models were obviously more accurate than the ones from $2 \mathrm{D}$ images. Thus, 3D modeling had a better advantage in the monitoring of
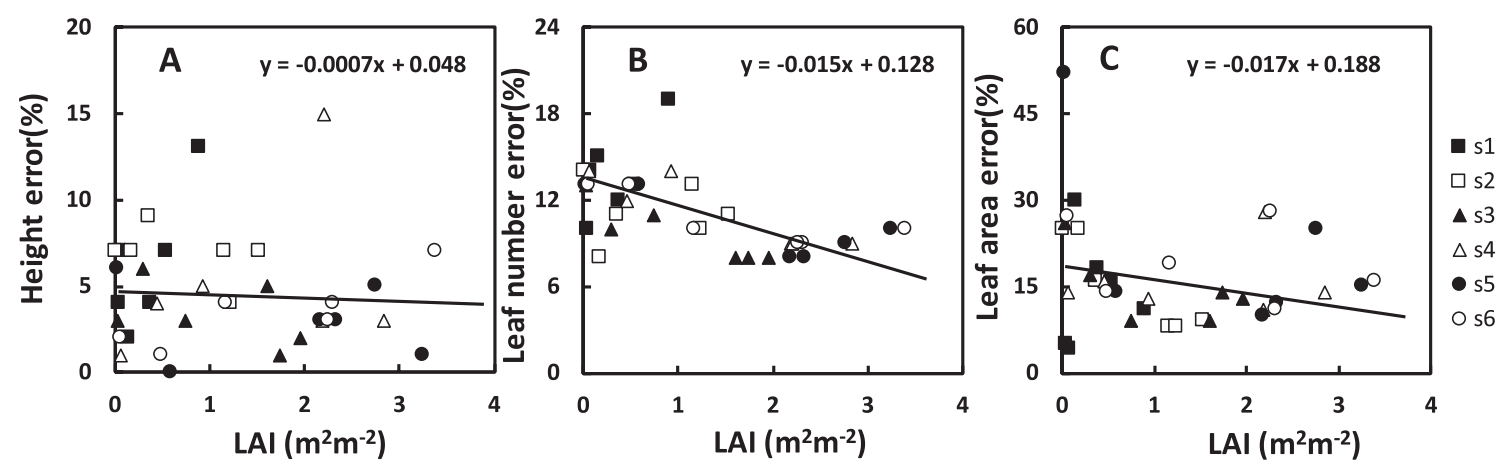

Fig. 7. The relationship between LAI and the percentage error of growth parameters estimated from 3D models in 6 sample blocks at different growth periods: (A) Plant height; (B) Leaf number; (C) Leaf area. 
plant growth and structure parameters.

As is seen above, 2D imaging had some problems, such as not being robust against occlusion of plant organs because of many overlapping leaves and stems, not being able to get fully $3 \mathrm{D}$ information because of complex 3D structure of plants, and the ambiguity between object size and distance from the camera. Meanwhile, as seen in Fig. 8, the growth parameters estimation from 3D models was reasonable results because of improvements of the above problems for 2D imaging.

\subsection{Estimation of growth parameters using regression models between the direct measured values and the values estimated from 3D model}

In the above section, we showed the growth parameters were directly estimated from $3 \mathrm{D}$ models within the reasonable error percentage. In this section, for more improvement of the estimates, we examined a method to calibrate the estimates from $3 \mathrm{D}$ models using the regression model between the measured value and the value estimated from 3D model. Figure 9 shows the regression models for plant height, leaf number and leaf area. The regression models were linear as $y=0.964 x+0.394\left(R^{2}=0.97\right.$ and $\mathrm{RMSE}=0.71 \mathrm{~cm})$ for plant height, $\mathrm{y}=1.091 \mathrm{x}+3.815\left(\mathrm{R}^{2}=0.99\right.$ and $\mathrm{RMSE}=4.03)$ for leaf number, and $\mathrm{y}=1.188 \mathrm{x}-0.015$ $\left(\mathrm{R}^{2}=0.98\right.$ and $\left.\mathrm{RMSE}=0.12 \mathrm{~m}^{2}\right)$ for leaf area, where $\mathrm{x}$ is the estimated value from $3 \mathrm{D}$ model described in the material method and $y$ is the measured value in Fig. 6 .

The results showed the very high regression for each parameter. As the result, the RMSE based on the regression models for plant height, leaf number and leaf area become very small as $0.71 \mathrm{~cm}, 4.03$ and $0.12 \mathrm{~m}^{2}$, respectively. In the experiment, we used data across a wide range in fertilizer supply and growth stages of sweet potato plants. So, in the conclusion, we confirmed the validity of the method to calibrate the estimates from 3D models using the regression model between the measured value and the value estimated from 3D model.

\subsection{The error analysis and accuracy assessments of measured parameters}

The measurements of plant growth and growth parameters are challenging, time consuming, and often rely on destructive methods. Now as 3D imaging technology is becoming a good solution for the monitoring and estimation of plant growth and structure parameters (Li et al., 2014; Zhang et al., 2016), 3D models allow accurate plant growth features estimation indoor and outdoor without the need to destructively cut off any parts of a plant. As an application of 3D imaging technology, some active methods and passive methods are widely used to construct 3D models for plants during the growth periods but they are constrained by the experiment environment and condition along with data acquisition and computational limitations.

In this study, we applied a novel 3D processing method based on SfM algorithms to reconstruct a fine-scale sweet potato plant model from multi-view images taken by a single SLR camera. We mainly analyzed the accuracy of plant growth and structure parameters at different fertilizer under 6 different periods and compared their performances of $3 \mathrm{D}$ shape reconstruction based on plant height, leaf number and leaf area. In our experiment design, the tested if sweet potato plants can be reliably detected with high accuracy and efficiency by our system platform. According to the experiments, we not only investigated the error between the estimated and measured values for plant height, leaf number and leaf area was small, but also investigated that the relationship between LAI and absolute error or percentage error with the fertilizer treatment. The measured parameter error correlates with the LAI of sweet potato plants in the test sample area during the different growth periods. The results in 3D models demonstrated that the errors linearly increased for plant height and leaf number and exponentially for leaf area with increasing in LAI, but the average percentage error shown in the regression line was a constant about $4.8 \%$ for plant height or decreased from $13 \%$ to $8 \%(\mathrm{LAI}=3.5)$ for leaf number and from $19 \%$ to $12 \%(\mathrm{LAI}=3.5)$ for leaf area with increasing in LAI, although each percentage error fluctuated, especially at low LAI. In contrast, the average percentage error in $2 \mathrm{D}$ image processing was $20 \%$ to $45 \%(\mathrm{LAI}=3.5)$ for leaf number and $60 \%$ to $90 \%$ $(\mathrm{LAI}=3.5)$ for leaf area, and the leaf height was immeasurable. Comparing with other studies, for example, Paproki et al. (2012) tested six small Gossypium hirsutum plants over four time-points indoor and made the parameter estimation for the retrieval of measurements such as main stem height and inclination, petiole length and initiation angle, and leaf width, leaf length and leaf area, and the observed errors ranging from $5.75 \%$ to $9.34 \%$. It was obvious that our performance was better than the percentage error $(9.34 \%)$ of plant height demonstrated by Paproki et al. (2012). And, it is point out that our results were obtained in the field experiments outdoor, instead of indoor. Besides, comparing
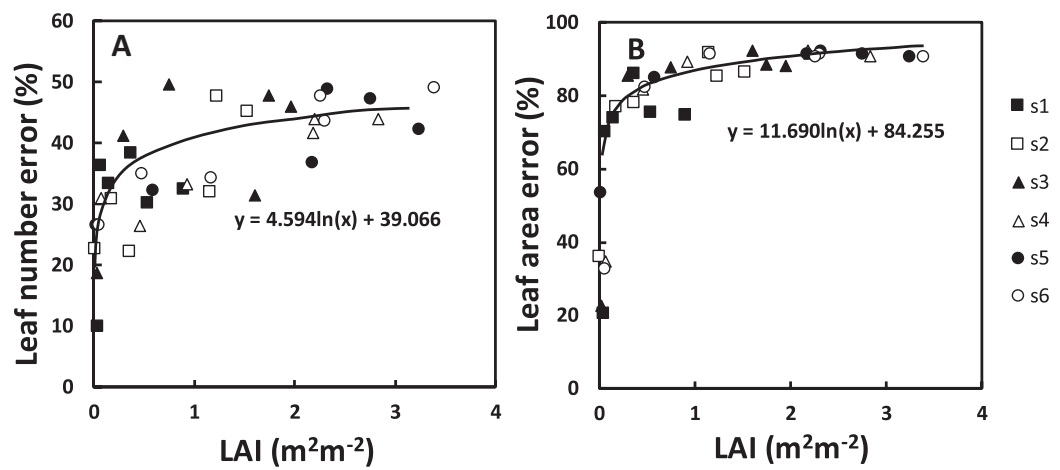

Fig. 8. The relationship between LAI and the percentage error of growth parameters estimated from 2D images in 6 sample blocks at different growth periods: (A) Leaf number; (B) Leaf area. 

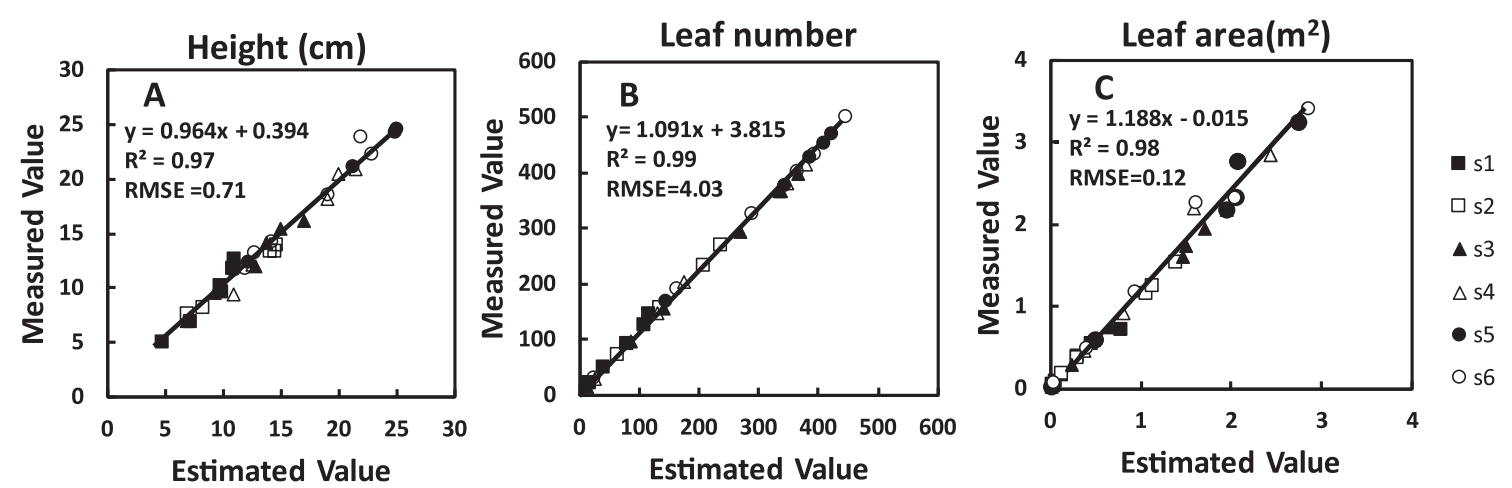

Fig. 9. The accuracy comparison of sweet potato plants growth parameters between measured value and estimated value in 6 sample blocks at different growth periods: (A) Plant height; (B) Leaf number; (C) Leaf area.

with the errors of $3 \mathrm{D}$ results, the errors of $2 \mathrm{D}$ images were much larger. The accuracy of predicting leaf area with this approach would certainly be dependent on the accuracy of the $3 \mathrm{D}$ models. On the whole, the experimental results with good fit between LAI and growth parameters error also confirmed the feasibility and validity of the proposed SfM methodology for an accurate estimation of plant parameters.

However, there are some errors or noises during the reconstructing 3D models of plant growth and structure parameters, especially for the measurement of leaf area, due partially to the errors inherent in field measurements and partially to the errors induced. For example, there were more re-projection errors for leaf in 3D model in S5 and S6 sample blocks according to the measurements in Fig. 6C. Errors in the leaf area were mainly caused by overlapping leaves. The errors were resulted from the overlapping of lush leaves, incorrect estimation of the feature points and color extraction in segmentation of surroundings such as other leaves and stems, grass, soil, plastic, etc. In order to reduce point noises and re-projection errors mentioned previously, it is necessary to improve camera calibration algorithm (e.g. distortion etc.) for increasing the search accuracy of target positions and solving the correspondence problem. As for the occluded leaves and wind-related problems, further improvement is needed to retrieve better leaf and plant structures, as well as setting fixed holder, increasing overlapping ratio, improving light conditions and so on.

Meanwhile, we also examined a method to calibrate the estimates from $3 \mathrm{D}$ models using the regression model between the measured value and the value estimated from $3 \mathrm{D}$ model. The regression model gave an improved coefficient of determination, and hence it made good estimations and could improve the image analysis method with capturing a linear relationship. According to the results, the regression models showed the linear and good estimation for leaf height $\left(\mathrm{R}^{2}=0.97\right.$ and $\mathrm{RMSE}=0.71 \mathrm{~cm})$, leaf number $\left(\mathrm{R}^{2}=0.99\right.$ and $\left.\mathrm{RMSE}=4.03\right)$ and leaf area $\left(\mathrm{R}^{2}=0.98\right.$ and $\left.\mathrm{RMSE}=0.12 \mathrm{~m}^{2}\right)$, in spite of use of data across wide range in fertilizer supply and growth stage of sweet potato plants. Comparing with other studies, for example, Rose et al. (2015) presented a photogrammetric approach for high accuracy measurement at the organ level of three-week-old tomato plants in the greenhouse and showed all structure parameters highly correlated with the reference measurements, yielding an $\mathrm{R}^{2}$ lying between $0.96-0.99$, including main stem height and leaf area. But the study did not consider the relationship between LAI and the error of growth parameters with the widely different fertilizer treatment and the whole growth stages. Jay et al. (2015) presented a method to retrieve row structure and structural parameters for 5 small plants, i.e., plant height and leaf area, and obtained two dataset collections during the two growth stages in the field. The two parameters were well estimated since high correlations and low average errors were obtained between estimated and actual values. However, their method did not consider occluded leaves and lacked further investigation to retrieve plant height and leaf area as well as LAI for such plant structure. According to our results, the performance was similar to the correlation of $\mathrm{R}^{2}$ over 0.96 demonstrated by Rose et al. (2015) and the estimation for leaf height $\left(R^{2}=0.99\right.$ and $\left.\mathrm{RMSE}=1.1 \mathrm{~cm}\right)$ and leaf area $\left(\mathrm{R}^{2}=0.94\right.$ and $\mathrm{RMSE}=0.0085 \mathrm{~m}^{2}$ ) shown by Jay et al. (2015). In our study in field, we made 6 dataset collections for sweet potato plants at the whole growth stages and wide different fertilizer conditions, and also investigated the structure parameters including plant height, leaf number and leaf area. Our results demonstrated that the error between the estimated and measured variables was small under the current experimental condition, whose correlation coefficient showed a fairly good agreement. The results have demonstrated that the regression model enabled our image analysis to estimate growth variables of sweet potato plants with a high degree of accuracy and flexibility. Therefore, our method and photography system, after being calibrated and improved by using the regression model, successfully identified the growth status of sweet potato plants with a high degree of estimation accuracy.

In sum, this study suggested that $3 \mathrm{D}$ imaging technique in the study has the further potential to remotely monitor plant growth status and estimate growth and structure parameters under various environmental factors outdoors. In the future experiments, improvements to the approach presented in this study would include the implementation of a physically-based model. Future work will also include the acquisition and analysis of multispectral and thermal imagery collected. A multi-camera approach could provide more detailed information about the growth of plants and the key components of the vegetation. In the future, such technology could be used to monitor farmland and agriculture production automatically in the field or greenhouse without the need for destructive sampling of these unique and fragile ecosystems. 


\section{Acknowledgments}

We gratefully acknowledge Mr. Yoshio Suzuki and the support staffs in the National Institute for Environmental Studies for the experiments.

\section{References}

Carrivick JL, Smith MW, Quincey DJ, 2016: Structure from Motion in the Geosciences, John Wiley \& Sons, Ltd, Chichester, UK. 1-208.

Clevers JGPW, Van Leeuwen HJC, 1996: Combined use of optical and microwave remote sensing data for crop growth monitoring. Remote Sensing of Environment 55, 42-51.

Gonzalez RC, Woods RE, 2008: Digital Image Processing $\left(3^{\text {rd }}\right.$ Edition). Peason Education. Prentice-Hall, New Jersey. 1-797.

Hosoi F, Nakabayashi K, Omasa K, 2011: 3-D modeling of tomato canopies using a high-resolution portable scanning lidar for extracting structural information. Sensors 11, 2166-2174.

Höhle J, Höhle M, 2009: Accuracy assessment of digital elevation models by means of robust statistical methods. ISPRS Journal of Photogrammetry and Remote Sensing 64, 398-406.

Harwin S, Lucieer A, 2012: Assessing the accuracy of georeferenced point clouds produced via multi-view stereopsis from unmanned aerial vehicle (UAV) imagery. Remote Sensing 4, 1573-1599.

Jay S, Rabatel G, Hadoux X, Moura D, Gorretta N, 2015: In-field crop row phenotyping from 3D modeling performed using structure from motion. Computers and Electronics in Agriculture 110, 70-77.

Kazmi W, Foix S, Alenya G, Andersen HJ, 2014: Indoor and outdoor depth imaging of leaves with time of flight and stereo vision sensors: Analysis and comparison. ISPRS Journal of Photogrammetry and Remote Sensing 88, 128-146.

Koniaris C, Cosker D, Yang XS, Mitchell K, 2014: Survey of texture mapping techniques for representing and rendering volumetric mesostructure. Journal of Computer Graphics Techniques 3, 18-60.

Lati RN, Manevich A, Filin S, 2013: Three-dimensional image-based modeling of linear features for plant biomass estimation. International Journal of Remote Sensing 34, 6135-6151.

Leberl F, Irschara A, Pock T, Meixner P, Gruber M, Scholz S, Weichert A, 2010: Point clouds: Lidar versus 3D vision. Photogrammetric Engineering \& Remote Sensing 76, 1123-1134.

Li L, Zhang Q, Huang D, 2014: A review of imaging techniques for plant phenotyping. Sensors 14, 20078-20111.

Lowe D, 2004: Distinctive image features from scale-invariant key points. International Journal of Computer Vision 60, 91-110.

Lou L, Liu Y, Sheng M, Han J, Doonan JH, 2014: A cost-effective automatic $3 \mathrm{D}$ reconstruction pipeline for plants using multi-view images. In: Advances in Autonomous Robotics Systems. TAROS 2014 (ed. by Mistry M, Leonardis A, Witkowski M, Melhuish C), Lecture Notes in Computer Science 8717, Springer, Cham 221-230.

Mathews AJ, Jensen JLR, 2013: Visualizing and quantifying vineyard canopy LAI using an unmanned aerial vehicle (UAV) collected high density structure from motion point cloud. Remote Sensing 5, 2164-2183.

Muller-Linow M, Pinto-Espinosa F, Scharr H, Rascher U, 2015: The leaf angle distribution of natural plant populations:
Assessing the canopy with a novel software tool. Plant Methods 11, 1-16.

Nguyen TT, Slaughter DC, Townsley B, Carriedo L, Maloof JN, Sinha N, 2016: Comparison of structure-from motion and stereo vision techniques for full in-field $3 \mathrm{D}$ reconstruction and phenotyping of plants: An investigation in sunflower. In: Proceedings of the ASABE Annual International Meeting. Orlando, Florida, USA.

Oliensis JA, 2000: Critique of structure-from-motion algorithms. Computer Vision and Image Understanding 80, 172-214.

Omasa K, 1990: Image instrumentation methods of plant analysis. In: Physical Methods in Plant Sciences (ed. by Linskens HF, Jackson JF), Modern Methods of Plant Analysis, Springer-Verlag, Berlin, Germany 11, 203-243.

Omasa K, Kouda M, 1998: 3-D color video microscopy of intact plants : A new method for measuring shape and growth. Environ. Control in Biol. 36(4), 217-226.

Omasa K, Hosoi F, Konishi A, 2007: 3D lidar imaging for detecting and understanding plant responses and canopy structure. Journal of Experimental Botany 58, 881-898.

Omasa K, Qiu GY, Watanuki K, Yoshimi K, Akiyama Y, 2003: Accurate estimation of forest carbon stocks by 3-D remote sensing of individual trees. Environmental Science \& Technology. 37, 1198-1201.

Paproki A, Sirault X, Berry S, Furbank R, Fripp J, 2012: A novel mesh processing based technique for $3 \mathrm{D}$ plant analysis. $B M C$ Plant Biology. 12, 1-13.

Richards JA, 2012: Remote sensing digital image analysis: an introduction. Springer Science \& Business Media. 1-494.

Rose JC, Paulus S, Kuhlmann H, 2015: Accuracy analysis of a multi-view stereo approach for phenotyping of tomato plants at the organ level. Sensors 15, 9651-9665.

Rovira-Más F, Zhang Q, Reid J, 2005: Creation of three-dimensional crop maps based on aerial stereo images. Biosystems Engineering 90, 251-259.

Santos TT, Koenigkan LV, Barbedo JGA, Rodrigues GC, 2014: 3D plant modeling: localization, mapping and segmentation for plant phenotyping using a single hand-held camera. In: Computer Vision - ECCV 2014 Workshops (ed. by Agapito L, Bronstein M, Rother C), Lecture Notes in Computer Science 8928, Springer, Cham, 247-263.

Se S, Pears N, 2012: Passive 3D imaging. In: 3D Imaging Analysis and Applications (ed. by Pears N, Liu Yh, Bunting P), Springer-Verlag London, 35-94.

Snavely N, Seitz SM, Szeliski R, 2006: Photo tourism: exploring photo collections in 3D. ACM Transactions on Graphics 25, 835-846.

Teng P, Zhang Y, Shimizu Y, Hosoi F, Omasa K, 2016: Accuracy assessment in $3 \mathrm{D}$ remote sensing of rice plants in paddy field using a small UAV. Eco-Engineering. 28, 107-112.

Tippetts B, Lee DJ, Lillywhite K, Archibald J, 2013: Review of stereo vision algorithms and their suitability for resource limited systems. Journal of Real-Time Image Processing 11, 5-25.

Turner D, Lucieer A, Watson C, 2012: An automated technique for generating georectified mosaics from ultra-high resolution unmanned aerial vehicle (UAV) imagery, based on structure from motion (SfM) point clouds. Remote Sensing 4, 1392-1410.

Yamamoto K, 2004: https://www.agr.nagoya-u.ac.jp/ shinkan/ LIA32/index-e.html

Zhang Y, Teng P, Shimizu Y, Hosoi F, Omasa K, 2016: Estimating 3D leaf and stem shape of nursery paprika plants by a novel multi-camera photography system. Sensors 16, 1-18. 